\title{
Lingeling Essentials \\ A Tutorial on Design and Implementation Aspects of the the SAT Solver Lingeling
}

\author{
Armin Biere \\ Institute for Formal Models and Verification \\ Johannes Kepler University, Linz, Austria \\ biere@jku.at
}

\begin{abstract}
One of the design principles of the state-of-the-art SAT solver Lingeling is to use as compact data structures as possible. These reduce memory usage, increase cache efficiency and thus improve runtime, particularly, when using multiple solver instances on multi-core machines, as in our parallel portfolio solver Plingeling and our cube and conquer solver Treengeling. The scheduler of a dozen inprocessing algorithms is an important aspect of Lingeling as well. In this talk we explain these design and implementation aspects of Lingeling and discuss new direction of solver design.
\end{abstract}

\section{References}

[1] A. Biere. Lingeling and friends at the SAT Competition 2011. Technical report, FMV Reports Series, Institute for Formal Models and Verification, Johannes Kepler University, Altenbergerstr. 69, 4040 Linz, Austria.

[2] A. Biere. PicoSAT essentials. JSAT, 4(2-4):75-97, 2008.

[3] A. Biere. Lingeling and friends entering the SAT Challenge 2012. In A. Balint, A. Belov, A. Diepold, S. Gerber, M. Järvisalo, and C. Sinz, editors, In Proceedings of SAT Challenge 2012: Solver and Benchmark Descriptions, volume B-2012-2 of Department of Computer Science Series of Publications B, pages 33 - 34. University of Helsinki, 2012.

[4] A. Biere. Lingeling, Plingeling and Treengeling entering the SAT Competition 2013. In A. Balint, M. J. H. Belov, A. Heule, and M. Järvisalo, editors, In Proceedings of SAT Competition 2013, volume B-2013-1 of Department of Computer Science Series of Publications B, pages 51 - 52. University of Helsinki, 2013.

[5] A. Biere. Yet another local search solver and Lingeling and friends entering the SAT Competition 2014. Technical report, 2014.

[6] M. Heule, M. Järvisalo, and A. Biere. Clause elimination procedures for cnf formulas. In C. G. Fermüller and A. Voronkov, editors, 17th International Conference on Logic for Programming, Artificial Intelligence, and Reasoning (LPAR'17), volume 6397 of Lecture Notes in Computer Science, pages 357-371. Springer, 2010.

[7] M. Järvisalo, M. Heule, and A. Biere. Inprocessing rules. In B. Gramlich, D. Miller, and U. Sattler, editors, 6th International Joint Conference Automated Reasoning (IJCAR'12), volume 7364 of Lecture Notes in Computer Science, pages 355-370. Springer, 2012. 